Hence $x y<6$, and $y(x+1) \equiv 0(\bmod 5)$. The solutions for $(x, y)$ are $(0,10),(16,0)$ and $(4,1)$. Only the last choice gives integral values for $f_{j}$ and we then have by (6.5) and (7.11),

$$
\begin{gathered}
\left(\tilde{\tau}_{\alpha j}^{\circ}\right)=\left\|\begin{array}{rrr}
1 & 1 & 1 \\
10 & -5 & 1 \\
16 & 4 & -2
\end{array}\right\|, \quad\left(\psi_{\alpha j}^{*}\right)=\left\|\begin{array}{ccc}
1 & 1 & 1 \\
1 & -1 / 2 & 1 / 10 \\
1 & 1 / 4 & -1 / 8
\end{array}\right\|, \\
f_{2}=\frac{27}{4.5}=6, \\
f_{3}=\frac{27}{1.35}=20 .
\end{gathered}
$$

The irreducible components have degrees $1,6,20$, and the characters may be found by applying (6.10).

Michigan State College

\title{
EQUAL SUMS OF LIKE POWERS
}

\section{E. M. WRIGHT}

Let $s \geqq 2$ and let $P(k, s)$ be the least value of $j$ such that the equations

$$
\sum_{i=1}^{j} a_{i 1}^{h}=\sum_{i=1}^{j} a_{i 2}^{h}=\cdots=\sum_{i=1}^{j} a_{i s}^{h} \quad(1 \leqq h \leqq k)
$$

have a nontrivial solution in integers, that is, a solution in which no set $\left\{a_{i u}\right\}$ is a permutation of another set $\left\{a_{i v}\right\}$. It was remarked by Bastien [1] ${ }^{1}$ that $P(k, 2) \geqq k+1$ and this is true $a$ fortiori for general $s$. The only upper bound for $P(k, s)$ for general $k$ and $s$ which I have found in the literature is due to Prouhet [5] who (in 1851) gave solutions of (1) with $j=s^{k}$, so that $P(k, s) \leqq s^{k}$. He allocates each of the numbers $0,1, \cdots, s^{k+1}-1$ to the set $\left\{a_{i u}\right\}$ if the sum of its digits in the scale of $s$ is congruent to $u(\bmod s)$. Recently Lehmer [4] took $m_{1}, \cdots, m_{k+1}$ any $k+1$ integers, let each of $b_{1}, \cdots, b_{k+1}$ run through 1947.

Presented to the Society, October 25, 1947; received by the editors September 23,

1 Numbers in brackets refer to the bibliography at the end of the paper. 
the values $0,1, \cdots, s-1$ and allocated the number

$$
b_{1} m_{1}+\cdots+b_{k+1} m_{k+1}
$$

to the set $\left\{a_{i u}\right\}$ if $\sum b_{l} \equiv u(\bmod s)$. Lehmer's method provides a solution which may be trivial, though any set of $m_{l}$ which makes the numbers (2) all different will certainly give a nontrivial solution. Prouhet's case, in which $m_{l}=s^{l-1}(1 \leqq l \leqq k+1)$, clearly does this.

The problem of determining $P(k, 2)$ has received much attention. The inequality $P(k, 2) \leqq 2^{k}$, a particular case of Prouhet's result, was rediscovered in 1912 by Tarry [6] and by Escott [8]. This has since been improved [7] to

$$
P(k, 2) \leqq\left(k^{2}+4\right) / 2 .
$$

In this note I find upper bounds for $P(k, s)$ for general $k$ independent of $s$ and comparable with (3). Unlike Prouhet I do not find a particular solution of (1), but my method gives bounds for the $a$. I cannot prove that $P(k, s)$ is independent of $s$, though I conjecture (somewhat more tentatively than for $P(k, 2)$ in [7]) that $P(k, s)$ $=k+1$.

Various authors $[2,3]$ have shown that $P(k, 2)=k+1$ for $1 \leqq k \leqq 9$ and Gloden [3] proved that $P(k, s)=k+1$ for $k=2,3$, and 5 and for all $s$.

Theorem 1. $P(k, s) \leqq\left(k^{2}+k+2\right) / 2$.

Let $j=\left(k^{2}+k+2\right) / 2, n=(s-1) j ! j^{k}, 1 \leqq a_{r} \leqq n(1 \leqq r \leqq j)$, and

$$
l_{h}=a_{1}^{h}+\cdots+a_{j}^{h}
$$

Then $j \leqq l_{h} \leqq j n^{h}$ and so there are at most

$$
\prod_{h=1}^{k}\left(j n^{h}-j+1\right)<j^{k} n^{k(k+1) / 2}
$$

different sets $l_{1}, \cdots, l_{k}$. But there are $n^{j}$ different sets $a_{1}, \cdots, a_{j}$ and so more than $j^{-k} n^{j-k(k+1) / 2}=(s-1) j$ ! sets $a_{1}, \cdots, a_{j}$ associated with some one set $l_{1}, \cdots, l_{k}$. Since the number of permutations of $j$ objects among themselves is $j$ !, there are at least $s$ sets $a_{1}, \cdots, a_{j}$ which have the same $l_{1}, \cdots, l_{k}$ and none of which is a permutation of any other. These provide a nontrivial solution of (1) with $1 \leqq a_{i u} \leqq(s-1) j ! j^{k}$.

THEOREM 2. If $k$ is odd, $P(k, s) \leqq\left(k^{2}+3\right) / 2$.

For $k=1$ the theorem is trivial. Let $k$ be odd, $k \geqq 3, m=(k-1) / 2$, 
$t=m(m+1)+1, n=(s-1) t ! t^{m}, 1 \leqq a_{r} \leqq n(1 \leqq r \leqq t)$, and

$$
L_{h}=a_{1}^{2 h}+\cdots+a_{t}^{2 h} \text {. }
$$

Since $t \leqq L_{h} \leqq t n^{2 h}$, the number of different sets $L_{1}, L_{2}, \cdots, L_{m}$ is at most

$$
\prod_{h=1}^{m}\left(t n^{2 h}-t+1\right)<t^{m} \prod_{h=1}^{m} n^{2 h}=t^{m} n^{t-1} .
$$

But there are $n^{t}$ different sets $a_{1}, \cdots, a_{t}$ and so more than $t^{-m} n(t !)^{-1}$ $=s-1$ sets $a_{1}, \cdots, a_{t}$ which have the same $L_{1}, \cdots, L_{m}$ and none of which is a permutation of any other. We take $s$ of these sets, denote the numbers in them by $a_{1}^{(u)}, \cdots, a_{t}^{(u)}(1 \leqq u \leqq s)$ and put

$$
\begin{array}{lr}
j=2 t=\left(k^{2}+3\right) / 2, & \\
a_{i u}=n+1+a_{i}{ }^{(u)} & (1 \leqq i \leqq t), \\
a_{i u}=n+1-a_{i-t}{ }^{(u)} & (t+1 \leqq i \leqq j)
\end{array}
$$

in (1). Since

$$
\sum_{i=1}^{j} a_{i u}^{h}=j(n+1)^{h}+2\left(\begin{array}{l}
h \\
2
\end{array}\right)(n+1)^{h-2} L_{1}+2\left(\begin{array}{l}
h \\
4
\end{array}\right)(n+1)^{h-4} L_{2}+\cdots
$$

and this is the same for all $u$ when $1 \leqq h \leqq k$, we have a nontrivial solution of (1).

\section{BIBLIOGRAPHY}

1. L. Bastien, Sphinx-Oedipe vol. 8 (1913) pp. 171-172.

2. L. E. Dickson, History of the theory of numbers, vol. 2, Washington, 1920, chap. XXIV.

3. A. Gloden, Mehrgradige Gleichungen, Groningen, Noordhoff, 1944.

4. D. H. Lehmer, Scripta Mathematica vol. 13 (1947) pp. 37-41.

5. M. E. Prouhet, C. R. Acad. Sci. Paris vol. 33 (1851) p. 225.

6. G. Tarry, L'intermédiaire des mathématiciens vol. 19 (1912) pp. 200, 219-221; vol. 20 (1913) pp. 68-70.

7. E. M. Wright, Quart. J. Math. Oxford Ser. vol. 6 (1935) pp. 261-267.

8. E. B. Escott, Quarterly Journal of Mathematics vol. 41 (1910) p. 145.

The University of AberdeEn 